\title{
Design of internal MRI coils using ultimate intrinsic SNR
}

\author{
Yiğitcan Eryaman · Yusuf Öner • Ergin Atalar
}

Received: 3 September 2008 / Revised: 7 February 2009 / Accepted: 26 February 2009 / Published online: 27 March 2009 (C) ESMRMB 2009

\begin{abstract}
Object Internal MRI coils have important applications in diagnostic and interventional studies. Since they can be placed very close to the region of interest in the body, they are favored over external coils in applications where high-resolution images are required. In this paper it is demonstrated that ultimate intrinsic SNR (UISNR) and the optimum coil sensitivity solutions can be used to make new coil designs with higher intrinsic SNR.

Materials and methods In this study, UISNR, which is the maximum attainable value of the intrinsic SNR, is used as a measure of performance and as a design criterion. As an example, a novel endorectal MRI coil is designed. The design is tested with phantom and patient studies.

Results An endorectal coil is built to demonstrate the effectiveness of the design strategy. ISNR of the endorectal coil approximates the UISNR to $72 \%$.

Conclusion An internal coil design method that takes advantage of the UISNR and optimum coil sensitivity calculations was presented. This method can also be used to design better internal MRI coils for different applications.
\end{abstract}

Keywords Internal MRI coils · Intrinsic SNR · Ultimate intrinsic SNR

\footnotetext{
Y. Eryaman $(\bowtie) \cdot$ E. Atalar

Department of Electrical and Electronics Engineering,

Bilkent University, 06800 Ankara, Turkey

e-mail: yigitcan@ee.bilkent.edu.tr

E. Atalar

e-mail: ergin@ee.bilkent.edu.tr

Y. Öner

Department of Radiology, Gazi University, Ankara, Turkey

e-mail: yusufoner@gazi.edu.tr
}

\section{Introduction}

MRI coils can be grouped into internal and external coils, in terms of the location of the coils with respect to the body. External coils receive the MR signal outside the human body, while internal coils are placed inside the body to obtain higher resolution images. There are many internal magnetic resonance imaging (MRI) coil types with different sizes and geometries. Intravascular [1,2], transesophageal [3], endourethral [4] and endorectal/transrectal coils [5,6] are designed for both diagnostic and interventional procedures. For each application, different design parameters should be considered. The main design parameters are the distance of the coil to the target tissue and the size and geometry of the internal coil. For most applications, maximization of the signal-tonoise ratio (SNR) of the acquired images is the main design problem. Although homogeneity may be considered a necessary requirement, signal intensity correction techniques [7,8] can be used to overcome this problem without further impacting underlying SNR of the image.

The SNR of an MR experiment depends not only on the performance of the coil but also on the imaging parameters. In order to separate coil performance-related components in the SNR from the imaging parameters, SNR is expressed in terms of intrinsic SNR (ISNR) [9] as:

$$
\mathrm{SNR}=\Psi \sqrt{t_{\mathrm{acq}}} \Delta V
$$

where $\Psi$ denotes the ISNR, $t_{\text {acq }}$ denotes the total data collection time of the MRI experiment and $\Delta V$ denotes the voxel volume. In order to optimize the performance of a receiver coil, the ISNR value obtained by the coil should be maximized in a given region of interest. The ISNR is shown in the expression below $[9,10]$ : 
$\Psi=\frac{\sqrt{2} \omega \mathrm{M}_{0}}{\sqrt{4 k_{\mathrm{b}} \mathrm{T} R}} B_{\mathrm{f}}$

where $T$ is the sample temperature, $k_{\mathrm{b}}$ is the Boltzmann's constant, $\omega$ is the Larmor frequency and $M_{0}$ is the equilibrium magnetization of the sample. $B_{\mathrm{f}}$ is the forward polarized magnetic field component and is defined as the complex number $B_{\mathrm{f}}=\left(B_{x}-\mathrm{i} B_{y}\right) / \sqrt{2}$, when the main magnetic field is along the $+z$ direction. $B_{x}$ and $B_{y}$ are the components of the magnetic field in the $x$ and $y$ directions. $R$ is the real part of the input impedance, seen by the input terminals of the coil. From the reciprocity principle [11], the value of $R$ can be found by calculating the total dissipated power when the receiver coil is used as a transmitter and driven by a $1 \mathrm{amp}$ rms current source from its input terminals. The signal and thermal noise expressions can be seen in the numerator and denominator, respectively, in Eq. 2.

In a previous work, ultimate intrinsic SNR (UISNR) for internal MRI coils was calculated as the upper limit of the ISNR of internal MRI coils [12]. The electromagnetic (EM) field inside a homogenous body model was expressed as a finite sum of cylindrical basis functions. These basis functions were the solutions for Maxwells's equations in cylindrical coordinates. Then, the EM field inside the body model was optimized in order to maximize the ISNR at a chosen point of interest. This model assumes that the coil is positioned in the inner region of the body and not close to a boundary, which is an acceptable assumption for most of the internal MRI coils. The UISNR was obtained by minimizing the value of $R$, the total dissipated power in the body, while keeping the forward polarized field component $B_{\mathrm{f}}$ constant at the point of interest. The UISNR is the maximum attainable ISNR in an MRI experiment, and it can be used as a measure of performance for internal MRI coils. Hence, it can be used as a figure of merit for the design process.

In an earlier work [13], a 4-channel phased array MRI coil configuration was designed and tested in both phantom and in vivo studies. The design strategy was based on obtaining the most appropriate placement of the coils for imaging the canine prostate with the highest SNR. The SNR data from the phantom experiments were compared to the UISNR for external coils. Coil performance maps (CPM) show the ratio of ISNR to UISNR. Since the UISNR for external coils does not set an upper bound for the ISNR of internal MRI coils, CPM values may exceed $100 \%$ in certain regions of the image showing that in those portions of the image, the ISNR of an internal coil is higher than that of the best possible external coil.

UISNR for external coils sets an upper bound for the ISNR of external MRI coils. Therefore, it can be used to compare the performance of certain internal MRI coil with respect to the optimum external MRI coil design. In this study, the design of internal MRI coils is based on approximating
UISNR for internal MRI coils, therefore, we aim to design the best performing internal MRI coil, rather than answering to the question whether the designed internal coil is better than the best possible external coil.

In this work, a new method of designing internal MRI coils is demonstrated, producing an endorectal coil. Experimental ISNR data obtained with this endorectal coil are compared to UISNR data to demonstrate its performance.

\section{Materials and methods}

For the design of the internal MRI coil, as well as any other receiver MRI coils, two coil parameters are crucial. $B_{\mathrm{f}}$, which is the forward polarized magnetic field component of the coil, and $R$, which is the real part of the input impedance of the coil. The coil of interest is a receive-only coil with possibly multiple channels. Both these parameters depend on the coil geometry and electromagnetic properties of the body. While designing an internal MRI coil, these two parameters must be considered. However, if this problem is not simplified with certain assumptions, it can be very complicated. In this paper, for the sake of simplicity, a heuristic approach is adopted, and the problem is divided into three sub-problems: choice of point of interest, cross-sectional geometry design and length optimization. Results obtained from each subproblem are used to design a final optimized coil. In order to demonstrate this idea, a new endorectal MRI coil design was made.

There are two constraints that should be considered for the design of endorectal coils. First, a suitable diameter for the internal coil is chosen. Hence, a tubular rigid structure is assumed for the coil geometry, since most of the body cavities are in this shape. The coil diameter should be chosen according to the application. For different diameters there may exist different optimum coil geometries. Second, while designing the coil, a primary concern was to obtain the maximum performance ratio, which is the ratio of the ISNR of the coil to the UISNR. Distribution of the performance ratio in the imaging plane is defined as the CPM. Since the value of the CPM of a coil depends on the radial distance from the coil center, a certain point of interest should be chosen. The radial distance of this point of interest to the coil center may vary depending on the application. Once the point of interest is chosen, the cross-sectional design and length optimization can be performed.

\section{Choice of point of interest}

Choosing an appropriate point of interest is the first step in designing an internal MRI coil. The farthest distance from the target organ, gland, etc. to the coil center should be chosen. The new coil design is intended to approximate the UISNR as 
much as possible at this certain point of interest. The distance of the point of interest is smaller than the wavelength therefore the optimum coil sensitivity exhibits a smooth monotonous decrease in the radial direction. Hence, it is guaranteed that the SNR of the resulting image will be higher everywhere on the target when compared to the point of interest. For this purpose, data regarding the dimensions of the target organ, and the distance of the farthest point to the coil center, should be collected from a number of patients. The mean value of the distance of the point of interest should then be calculated along with its standard deviation.

\section{Cross-sectional geometry design}

Cross-sectional coil geometry design is the second step of the design process. By assuming a constant $B_{\mathrm{f}}$ at the point of interest, an optimum field solution that gives the UISNR should be calculated. For these calculations, cylindrical body model with uniform EM properties can be used, since the distance of the point of interest is usually smaller than the wavelength for most of the internal coil applications. Once the optimum field solution is calculated, as the reciprocity principle suggests, a coil with a sensitivity that approximates the optimum coil sensitivity should be designed. Polarization and the angular variation of the optimum coil sensitivity should also be taken into account in this step. To understand the polarization of the optimum coil, the $B_{\mathrm{f}} / B_{\mathrm{r}}$ ratio can be evaluated at the point of interest. $B_{\mathrm{r}}$ is the reverse polarized magnetic field component of the optimum field, and $B_{\mathrm{r}}=\left(B_{x}+\mathrm{i} B_{y}\right) / \sqrt{2}$. If $B_{\mathrm{f}} / B_{\mathrm{r}} \approx 1$, this result automatically reveals that the optimum coil has a linear polarization, which can be implemented using a single loop geometry. However, for the cases $B_{\mathrm{f}} / B_{\mathrm{r}} \gg 1$, it will be necessary to use a multichannel coil configuration. Hence, the cross-sectional geometry of the coil must be modified by increasing the number of loop coils. On the other hand, for any angular variation in the optimum field sensitivity around the cavity, certain modifications should be made in the coil design in order to realize the optimum field distribution as much as possible. These modifications may include the addition of strips, loops or any other modifications that enable the control of the angular variation of the sensitivity.

The modification of the cross-sectional coil geometry with respect to optimum field calculations can be performed either by using computer simulations and/or phantom studies. On the other hand, the ISNR variation of the coil designs must be compared to the UISNR value for internal MRI coils. CPM should also be generated.

\section{Length optimization}

For the length optimization, the variation of the coil performance, with respect to coil length, should be considered. For this purpose, rectangular loop coils with different lengths can be simulated or experimentally tested. Other shapes such as an ellipse can also be tested if they increase the CPM. To obtain a uniform sensitivity in the $z$ direction, a rectangular loop shape is chosen. The CPM value of the coils with different length at the point of interest should be compared to each other. The coil length that gives the maximum CPM at the point of interest is the optimum coil length for that specific geometry.

\section{Coil implementation}

All materials including the capacitors, wires, connectors and coil housing should be non-magnetic. The matching-tuning should be performed and the receiver coil should be decoupled from the body coil of the scanner at Larmor frequency. For decoupling of individual channels of the coil, the twoport s21 parameter should be measured using a network analyzer.

\section{Results}

Coil diameter was chosen as $25 \mathrm{~mm}$ since this is close to the maximum tolerable endorectal probe diameter for most of the patients. The choice of the point of interest is crucial and may vary due to the application. For endorectal imaging, the prostate is the main gland to be imaged. The point of interest should be chosen such that the whole prostate will be covered in the axial sense. To achieve this, the farthest point of the prostate is chosen as the point of interest for coil design. If another location was chosen as the point of interest, such as the closest point of the prostate to the rectum wall, then the results could fail to give high SNR values at the farther regions of the prostate. Although the coil would be optimized to give the highest ISNR at the point of interest, it would not guarantee an acceptable ISNR at other locations of the prostate gland. Hence, the farthest point of the prostate to the rectum wall must be considered for this design problem. For this purpose, prostate images from 12 patients, obtained with the same size endorectal coils, were analyzed. The distance between the farthest point of the prostate and the coil center was measured. Figure 1 shows the prostate image and the distance measurements made on this image. Four points, called X1, X2, X3 and X4, that define the borders of the prostate are marked on the image. Table 1 shows the mean value and standard deviation of the distance measurements obtained from these prostate images. The mean value of these measurements, $4.5 \mathrm{~cm}$ was obtained as the distance between the farthest point of the prostate and the coil center. Therefore, this distance is assumed as the most appropriate point of interest for the design of a new endorectal MRI coil. 


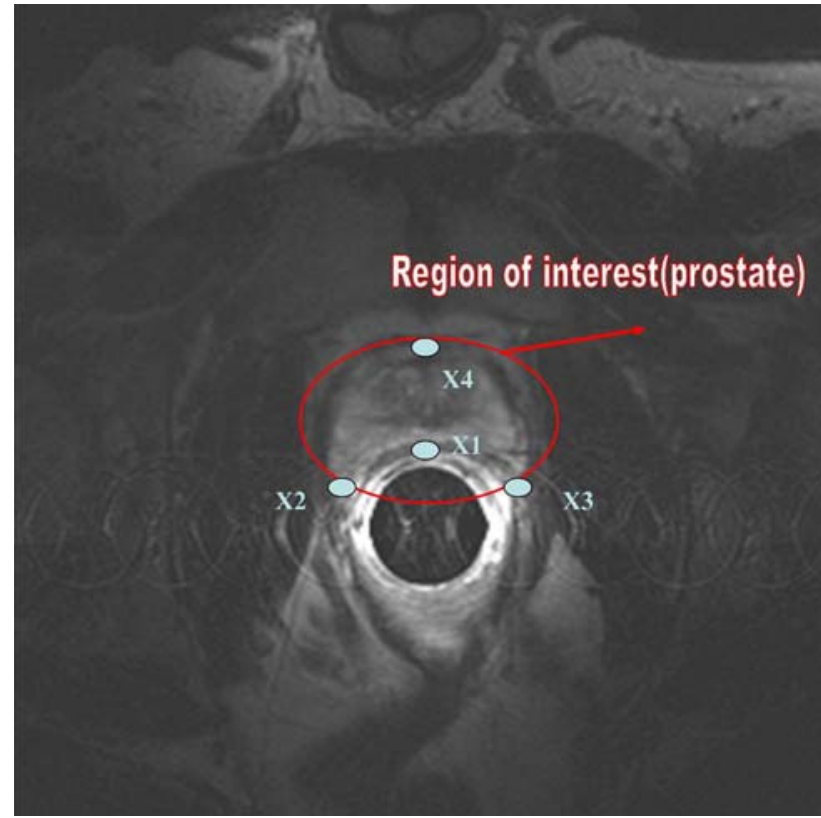

Fig. 1 A prostate image [courtesy of Peter Guion (NIH)] obtained by an endorectal MRI coil [6]

Table 1 Mean and standard deviation of the distances of spots X1, X2, $\mathrm{X} 3$ and $\mathrm{X} 4$ to the coil center

\begin{tabular}{lll}
\hline & Mean $(\mathrm{mm})$ & Standard deviation $(\mathrm{mm})$ \\
\hline $\mathrm{X} 1$ & 14.4 & 1.24 \\
$\mathrm{X} 2=\mathrm{X} 3$ & 25.1 & 3 \\
$\mathrm{X} 4$ & 44.8 & 4.3 \\
\hline
\end{tabular}

Borders of the prostate are marked on the image using 4 spots

For the cross-sectional geometry of the coil, an image of optimum sensitivity is generated. The coil geometry is designed in order to mimic the optimum sensitivity profile as much as possible. Figure 2 shows the optimum sensitivity profile. On the other hand, Fig. 3 shows the $B_{\mathrm{f}} / B_{\mathrm{r}}$ ratio sampled in the direction where the sensitivity, $B_{\mathrm{f}}$, is maximum.

Optimum sensitivity is obtained by solving the UISNR problem for the point of interest and mapping the $B_{\mathrm{f}}$ field in the axial plane in the vicinity of the cavity. As seen in Fig. 2, clearly there is an angular variation in the field. Since the internal coil is assumed to be located inside this cavity, and the point of interest is a single point, the field is directed toward that point in order to illuminate it effectively. If the opposite side of the cavity was also illuminated, the noise value would increase due to the excess electric field distribution. This field distribution does not contribute to the sensitivity in the vicinity of the point of interest. Hence, an asymmetrical field distribution is much more advantageous in order to obtain higher ISNR values at the point of interest. Figure 4 shows the single-channel strip-conductor coil, designed in order to achieve such a field distribution with

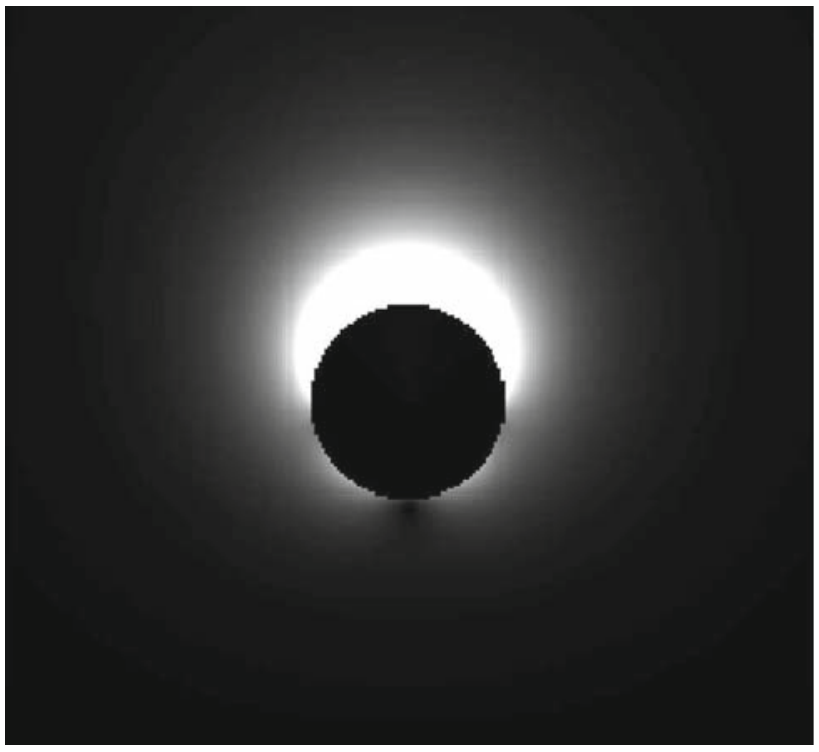

Fig. 2 Optimum coil sensitivity obtained by the UISNR calculations

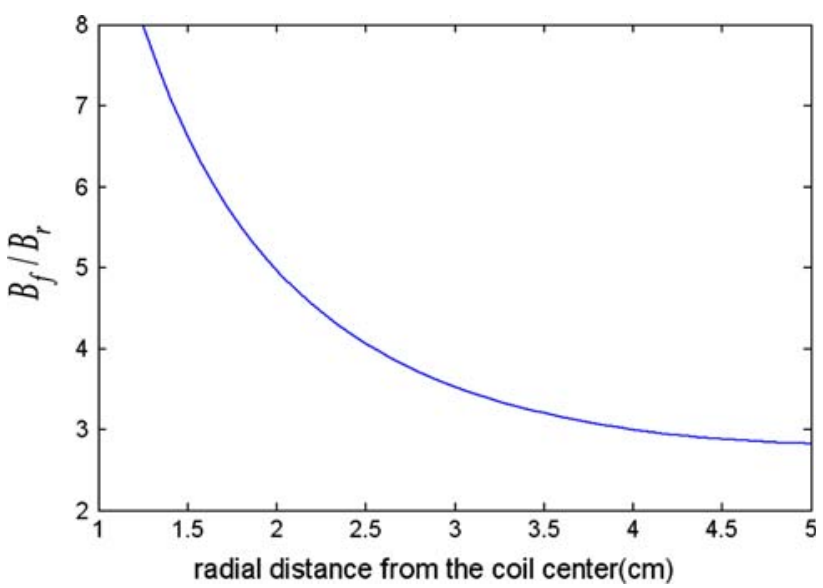

Fig. $3 B_{\mathrm{f}} / B_{\mathrm{r}}$ ratio sampled in the direction where $B_{\mathrm{f}}$ is maximum. Since $B_{\mathrm{f}} / B_{\mathrm{r}}>1$, the optimum coil should be composed of a quadrature structure

angular variation. This design has a semi-cylindrical copper strip used for one of the poles of its current loop. The strip causes the current to flow in a distributed manner and hence, the sensitivity at the strip side of the coil is canceled out. It is intended to reduce the noise due to the electric field distribution around the coil. With this design, a maximum improvement of $35 \%$ is achieved at a radial distance of $2.5 \mathrm{~cm}$. On the other hand, the second design, which is shown in Fig. 4, is a dual phased array coil. Figure 3 shows that the $B_{\mathrm{f}} / B_{\mathrm{r}}$ ratio changes between 8 and 2.9. Therefore, it is obvious that the optimum coil will not be composed of a single loop structure. Hence, a quadrature structure, as shown in Fig. 4b, is expected to give an ISNR improvement at the point of interest. This result is intuitive since, as the number of channels with uncorrelated noise is increased in 

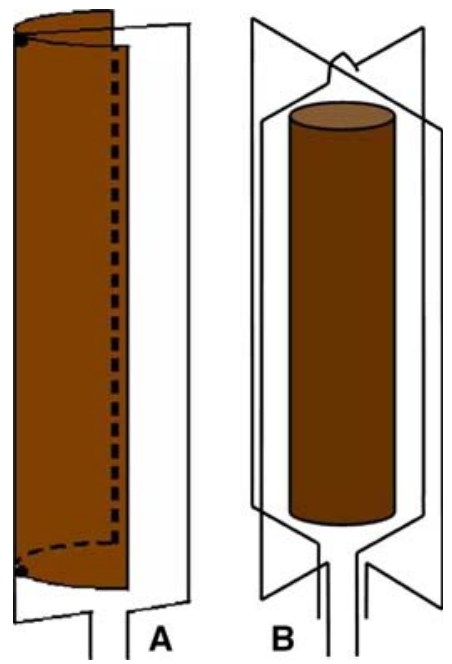

Fig. 4 Drawings of two coil geometries that give an SNR improvement over a conventional rectangular loop endorectal coil. The strip conductor coil (a) gives a maximum improvement of $35 \%$ at $2.6 \mathrm{~cm}$. The dual phased array coil (b) gives a maximum improvement of 55\% at $3.5 \mathrm{~cm}$ away from the coil center

a coil design, an improvement in ISNR is expected. The copper cylinder located in the middle of the two loops is used for decoupling of the two channels of the coil. The maximum improvement over a conventional loop coil was $55 \%$ with this coil, and it was obtained at a radial distance of $3.2 \mathrm{~cm}$ away from the coil center.

The ISNR variations of the coils shown in Fig. 4 are compared to the UISNR for internal coils, and it was seen that a maximum CPM value of $57 \%$ is obtained by the dual phased array coil at a distance of $6 \mathrm{~cm}$ away from the coil center. At the point of interest of $4.5 \mathrm{~cm}$, the CPM of the dual phased array coil drops to $45 \%$. Although these designs give an improvement over a conventional loop coil, CPM results show that they are still not optimum and their ISNR can be improved. For this purpose, the geometrical properties of the first two designs that resulted in an SNR improvement are combined in a single design, and a third design is proposed. Figure 5 shows this design.

In the designs shown in Figs. 4 and 5, in order to obtain uncorrelated noise between two channels, the two loop coils are decoupled with additional methods. There are many different methods to decouple receiver MRI coils in the literature $[4,13]$. One of the techniques used in this work for decoupling was placing an inner copper cylinder between the loops. Another method was to use a strip conductor, which also serves to modify the coil sensitivity, in order to obtain a field distribution with an angular variation. Among these two methods, the strip-conductor is more efficient. Furthermore, it can be used alone without the inner copper cylinder. The shape of the copper strip was modified in order to change the path of the current that flows on the loop. By proper
Fig. 5 A third design proposed to obtain further improvement in comparison to first two designs. Geometrical properties of the strip-conductor and dual phased array designs that resulted in SNR improvements are combined into a single design

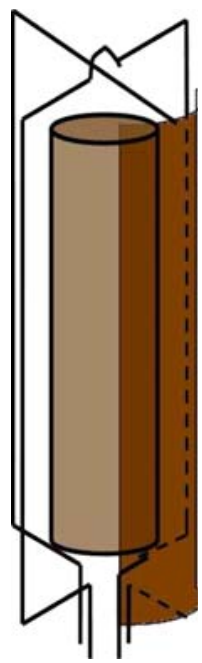

modification of the shape of the copper strip, the currents on two loops can be forced to flow in paths perpendicular to each other. As a measure of decoupling, the $\mathrm{s} 12$ parameter of the coil is measured using a two-channel network analyzer. $\mathrm{s} 12$ is minimized to $-22 \mathrm{~dB}$ with this method. This shows that by changing the shape of the copper strip the coupling between the two loops can be reduced significantly. The s11 and s22 parameters are minimized by means of appropriate matching and tuning. Reflection coefficients seen from both channels were reduced to a value less than 0.1 .

For the length optimization, rectangular loop coils with lengths varying between 2 and $12 \mathrm{~cm}$ are simulated by using the simulation software FEKO (EM Software and Systems Stellenbosch, SA). The ISNR values for each coil are calculated and then divided by the UISNR value corresponding to the same coil model. The results are shown in Fig. 6. As shown, there exists a different optimum coil length for different points of interests. For the point of interest with a radial

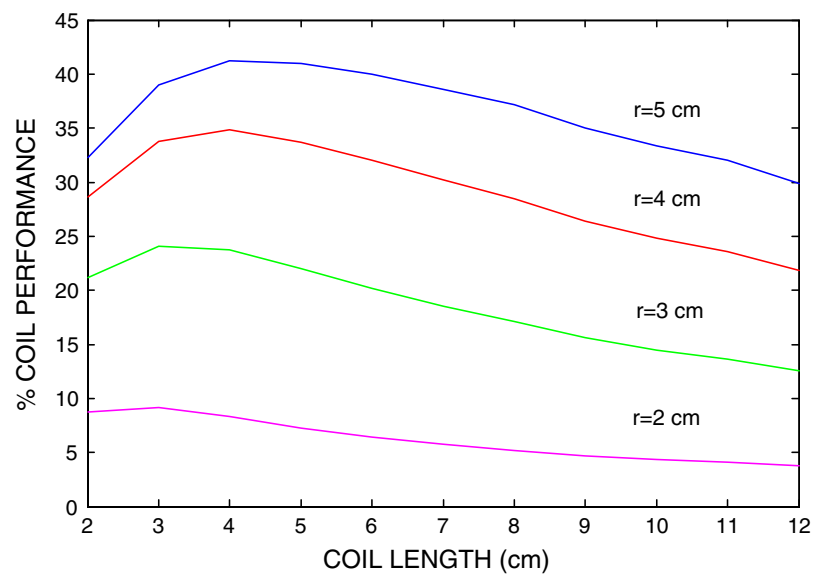

Fig. 6 Coil performance curves for different points of interests to be imaged. It can roughly be stated that $4 \mathrm{~cm}$ is the optimum coil length for all points of interests 
Fig. 7 Optimized endorectal coil

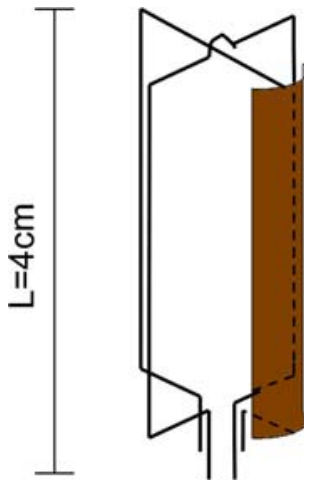

distance of $4.5 \mathrm{~cm}$ away from the coil center, the length of the optimized coil should be chosen as $4 \mathrm{~cm}$. By using the results obtained from the cross-sectional and length optimization, an optimized design can be proposed. Figure 7 shows a simple drawing of the optimized endorectal coil.

\section{Phantom studies}

A fast spin echo sequence on the GE 1.5 T Signa system was used with the following imaging parameters: FOV: $16 \mathrm{~cm}$; slice thickness $=3.5 \mathrm{~mm} ; \mathrm{BW}=41.6 \mathrm{kHz} ; \mathrm{TR}=10,000 \mathrm{~ms}$; $\mathrm{TE}=7.7 \mathrm{~ms}$. Axial images of a salt water phantom were obtained. The conductivity of the salt water solution was measured as $0.35 \mathrm{~S} / \mathrm{m}$. A performance map was obtained by dividing the ISNR variation of the optimized coil by the UISNR. Figure 8 shows the performance map of the optimized coil. CPM of the final design shows that the coil can approximate the UISNR by $85 \%$ at $6 \mathrm{~cm}$; however, this is not our target point. On the point of interest, the performance is $72 \%$. The angular CPM variation due to asymmetrical field

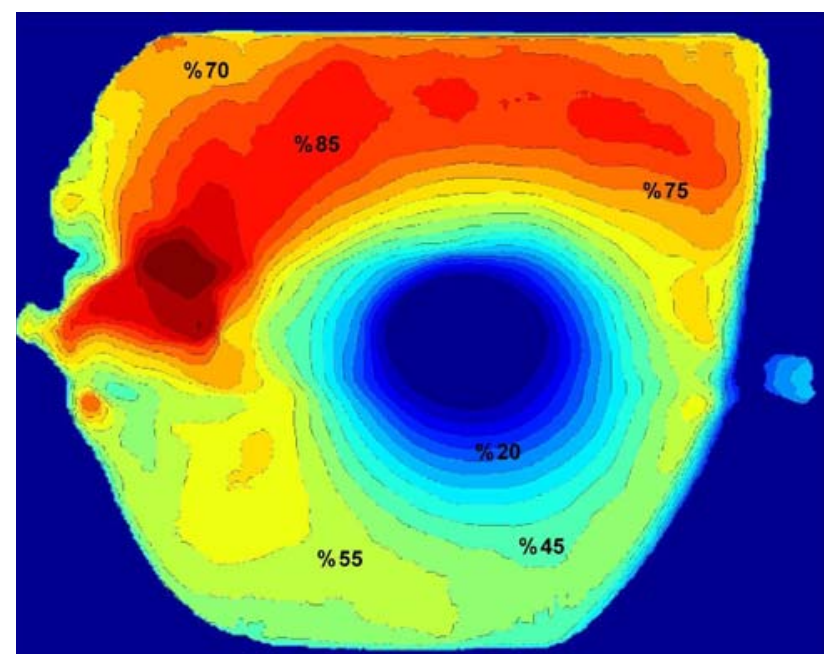

Fig. $8 \mathrm{CPM}$ of the optimized coil (FOV $16 \mathrm{~cm} \times 16 \mathrm{~cm}$ ). As shown, a maximum performance of $85 \%$ is obtained at a distance of $6 \mathrm{~cm}$ away from the coil center. At $4.5 \mathrm{~cm}$, the performance the coil is $72 \%$ distribution is also clear in the figure. The locations near the copper-strip have lower CPM values.

\section{Patient studies}

In order to demonstrate the clinical usability of the optimized coil, it was used in a patient study. For patient experiments, a few minor modifications were made in the design. A coil housing of $2.6 \mathrm{~cm}$ diameter and $15 \mathrm{~cm}$ length was built. For patient comfort, a flexible plastic material was chosen as the housing material. To image the prostate properly, the coil must be inserted into the inner regions of the rectum, and, therefore, the position of the sensitive region of the coil should be very close to the distal end of the coil. For this purpose, two coaxial lines were added between the loops and the matching and tuning circuitry. Since the coaxial line is very short when compared to wavelength and has low loss, the effect of this modification on the noise resistance is expected to be small. The loss due to this modification is calculated by using transmission line theory as 11\%. [14] Experiments were conducted on a GE Signa 1.5 system. Figure 9 shows the sensitivity corrected axial image of a 65-year-old patient's prostate obtained with this coil. A T2-weighted FRFSE sequence with parameters of $\mathrm{FOV}=16 \mathrm{~cm} \quad \mathrm{TE}=119 \mathrm{~ms}, \mathrm{TR}=2,900 \mathrm{~ms}, \mathrm{BW}=25 \mathrm{kHz}$ was used to obtain the images. A sensitivity correction technique was applied to the axial image, by assuming a $1 / r^{2}$ variation for the sensitivity, where $r$ is the radial distance from the center of the coil. The location of the center of the coil was estimated manually by finding the pixels that showed fast signal decay in the body-coil interface. In order to eliminate the errors due to the coil center estimation, the correction was not carried out in the immediate vicinity of the coil. In addition, in order to minimize the noise amplification, the correction was turned off far away from the coil.

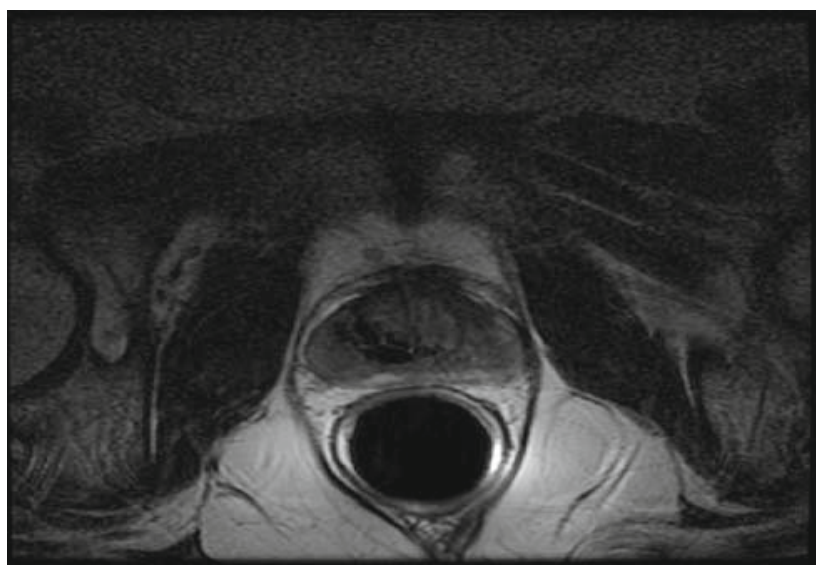

Fig. 9 Axial image of a prostate obtained with the optimized endorectal coil by using a T2-weighted FRFSE sequence with imaging parameters; FOV $=16 \mathrm{~cm} \times 16 \mathrm{~cm} \mathrm{TE}=119 \mathrm{~ms}, \mathrm{TR}=2,900 \mathrm{~ms}, \mathrm{BW}=25 \mathrm{kHz}$ 
This is accomplished by using the expression:

$$
\frac{\left(r / r_{\mathrm{n}}\right)^{2}+1}{\left(r / r_{\mathrm{f}}\right)^{2}+1}
$$

where $r_{\mathrm{n}}$ and $r_{\mathrm{f}}$ are the radii of the near and far region. The correction is carried out between $r_{\mathrm{n}}$ and $r_{\mathrm{f}}$.

The original image is divided by the expression above to obtain a more uniform sensitivity.

The values of $r_{\mathrm{n}}$ and $r_{\mathrm{f}}$ were chosen as 1.4 and $8.4 \mathrm{~cm}$, respectively, using trial and error.

\section{Discussion}

The main purpose of this study is to introduce a new coil design method based on UISNR calculations which focuses the technical development in the internal MRI coils, in general. The ISNR and the CPM at a given point of interest were the main parameters to be considered for optimization. The results regarding ISNR and CPM are demonstrated with phantom studies. The endorectal coil which was designed according to the proposed method has a CPM of $72 \%$ at the point of interest of $4.5 \mathrm{~cm}$. A coil design that precisely realizes optimum field distribution is expected to give a CPM of $100 \%$. However the implementation of such a coil is a very difficult task and requires the solution of complex inverse problems. Instead an easier approach was adopted. Modifications based on educated guesses which enabled a rough realization of the optimum field distribution were made. Since this realization is not precise, the results deviated from the maximum CPM of $100 \%$ at the point of interest.

The number of channels for the optimized design (Fig. 6) could be increased in order to obtain higher CPM but this was not the preferred; due to limited space, decoupling the channels separated by angles different than $90^{\circ}$ is a difficult task. The addition of more channels may increase ISNR, however, the obtained ISNR value is sufficiently close to UISNR. As an engineering judgement it was decided that no additional channels are needed.

The CPM of the optimized coil was demonstrated using images from a uniform phantom model. Finally, in order to demonstrate that the design is clinically usable a patient study was performed (Fig. 9). It is almost impossible to obtain true CPM results using patient studies since the tissue characteristics weight the overall image according to anatomical structure. On the other hand, the overall performance of the optimized coil was already demonstrated with phantom studies.

Due to length optimization, the optimum dimension of the coil was found as $4 \mathrm{~cm}$ according to the simulations. So, the new coil was built with a length of $4 \mathrm{~cm}$, and its performance variation was calculated using the results of the phantom study. The matching and tuning circuitry was located right at the end of the coil loops for the phantom tests in order to minimize the mismatch losses. For the patient studies, another coil was built, but this time, the coil loops were placed at the far end of the coil housing in order to cover the prostate correctly. To build the coil easily, the matching and tuning circuitry of the coil loops was placed outside the human body. The loops were connected to the matching and tuning circuitry via two coaxial lines inside the coil housing. This type of connection can result in a slight SNR loss if the coaxial cables are lossy. However, the coaxial cables were very short compared to the wavelength, and, since the coil dimension was large, thick low loss RG-58 coaxial cables were used for these connections. In order to avoid the SNR loss completely, the matching and tuning circuitry can be placed right after the coil loops so that the noise due to multiple reflections is zero.

In our calculations, we assume that the body cavity is along the $z$ direction. In the body there may be cases where the cavity is not aligned with the $z$ direction. The optimum internal coil design for this may be significantly different than the optimum design for $z$ directed cavity. However, our proposed design methodology is still valid. In this case UISNR calculations and the optimum coil sensitivity will change and therefore the corresponding optimum coil can be found.

\section{Conclusions}

In this paper, a design method for internal MRI coils was explained. To simplify the problem, a heuristic approach was adopted and the design problem was divided into three sub-problems: choice of point of interest, cross-sectional geometry design and length optimization. Results obtained from each sub-problem are used to design a final optimized coil. The effectiveness of the method is demonstrated by implementing a novel endorectal coil and testing it in phantom studies. In order to demonstrate that the coil is clinically usable patient studies were performed. The new design approximated the UISNR, which is the theoretically maximum attainable ISNR at the point of interest. Similar methods can be used to design different types of internal MRI coils.

\section{References}

1. Hurst GC, Hua J, Duerk JL, Cohen AM (1992) Intravascular (catheter) NMR receiver probe Preliminary design analysis and application to canine iliofemoral imaging. Magn Reson Med 24(2): 343-357

2. Ogan O, Atalar E (1997) Intravascular magnetic resonance imaging using a loopless catheter antenna. Magn Reson Med 37(1): $112-118$

3. Shunk KA, Lima JAC, Heldman AW, Atalar E (1999) Transesophageal magnetic resonance imaging. Magn Reson Med 41(4): 722726 
4. Quick HH, Serfaty J-M, Pannu HK, Genadry R, Yeung CJ, Atalar E (2001) Endourethral MRI. Magn Reson Med 45(1): 138-146

5. Schnall M, Lenkinski R, Pollack H, Imai Y, Kressel H (1989) Prostate: MR imaging with an endorectal surface coil. Radiology 172(2): 570-574

6. Krieger A, Susil RC, Menard C, Coleman JA, Fichtinger G, Atalar E, Whitcomb LL (2005) Design of a novel MRI compatible manipulator for image guided prostate interventions. IEEE Trans BME 52(2):306-313

7. Meyer CR, Bland PH, Pipe J (1995) Retrospective correction of intensity inhomogeneities in MRI. IEEE Trans Med Imaging 14(1): $36-41$

8. Sled JG, Zijdenbos AP, Evans AC (1998) A nonparametric method for automatic correction of intensity nonuniformity in MRI data. IEEE Trans Med Imaging 17(1): 87-97

9. Edelstein WA, Glover GH, Hardy CJ, Redington RW (1986) The intrinsic signal-to-noise ratio in NMR imaging. Magn Reson Med 3(4): 604-618
10. Ocali O, Atalar E (1998) Ultimate intrinsic signal-to-noise ratio in MRI. Magn Reson Med 39(3): 462-473

11. Hoult DI, Richards RE (1976) The signal-to-noise ratio of the nuclear magnetic resonance experiment. J Magn Reson Imaging 24: 71-85

12. Celik H, Eryaman Y, Altintas A, Abdel-Hafez IA, Atalar E (2004) Evaluation of internal MRI coils using ultimate intrinsic SNR. Magn Reson Med 52(3): 640-649

13. Yung AC, Öner AY, Serfaty J-M, Feneley M, Yang X, Atalar E (2003) Phased-array MRI of canine prostate using endorectal and endourethral coils. Magn Reson Med 49(4): 710-715

14. Pozar DM (1998) Microwave engineering. Wiley, NY, pp 94 